\title{
A Variable Background Active Contour Model for Automatic Detection of Thyroid Nodules in Ultrasound Images
}

\author{
M. Savelonas, D. Maroulis, D. Iakovidis \\ Dept. of Informatics and Telecommunication \\ University of Athens \\ Athens, Greece \\ rtsimage@di.uoa.gr
}

\author{
S. Karkanis \\ Dept. of Informatics and Computer Technology \\ Technological Educational Institute of Lamia \\ Lamia, Greece
}

\author{
N. Dimitropoulos \\ Dept. of Medical Imaging \\ EUROMEDICA Medical Center \\ Athens, Greece
}

\begin{abstract}
A novel active contour model named Variable Background Active Contour model is proposed and applied for the detection of thyroid nodules in ultrasound images. The new model offers edge independency, no need for smoothing, ability for topological changes and it is more accurate when compared to the Active Contour Without Edges model. Improved accuracy is achieved by introducing as background a limited image subset which appropriately changes shape to reduce the effects of background inhomogeneity. We validated the proposed model on ultrasound images acquired from 24 patients and the results demonstrate an improvement in accuracy when compared to the Active Contour Without Edges model.
\end{abstract}

\section{INTRODUCTION}

Thyroid nodules are abnormal lumps growing within the thyroid gland which may represent various different conditions including cancer. A non-invasive low-cost imaging technique widely used for the detection and the ascertainment of the size and number of thyroid nodules is ultrasonography [1].

The ultrasound images produced by this technique contain echo perturbations and speckle noise, which follows a Rayleigh distribution that cannot be modeled [2]. Thyroid nodule detection in such images imposes the use of a segmentation method that takes into consideration their inherent noise characteristics.

Two main classes of image processing methods exist for feature based image segmentation: region growing methods [35] and active contours [6-9]. Region growing methods include the definition of an initial set of seed pixels and the growing of a uniform and connected region from each seed according to an appropriately selected homogeneity criterion. Their main advantage is that they are insensitive to local perturbations as they test the statistics inside the region. However, they often generate irregular boundaries and small holes [6]
Early approaches of active contour models utilize local filtering techniques such as edge detection operators. These approaches are considered inappropriate for ultrasound images as the strong isotropic smoothing Gaussian that needs to be applied for noise reduction, introduces the risk of smoothing the edges corresponding to the object boundaries [7]. Efforts have been made towards the unification of contour and regionbased approaches [8, 9]. An improved region based active contour model that incorporates previous improvements was proposed by Chan and Vesse [7]. This model, named Active Contour Without Edges is based on the level set method [10] and the Mumford-Shah segmentation techniques [11] to build the stopping criterion for the curve evolution on the desired boundary instead of an edge function. This way the input image does not have to be smoothed, even if it contains noise and therefore the object boundaries are preserved and could be accurately detected. The Active Contour Without Edges model allows the detection of objects whose boundaries are either smooth or not necessarily defined by gradient. For both these cases the classical active contour models are not applicable. In addition, the level set formulation of this model enables the algorithm to detect two or more objects in the image as it provides adaptability to topological changes e.g. contour splitting. A limitation of the Active Contour Without Edges model is that it presumes homogeneity for object and background areas. A modification of this model taking into account image inhomogeneity could lead to more accurate object detection.

In this paper, we propose a novel active contour model that uses a variable background to reduce the effects of image inhomogeneities. This model aims to the enhancement of the object detection accuracy. To the best of our knowledge active contour models have not been employed for the accurate detection of thyroid nodules in ultrasound images. Thus we 
apply the proposed model as well as Active Contour Without Edges model for this application.

The rest of this paper is organized in three sections. Section II includes a brief description of the Active Contour Without Edges model and the presentation of the proposed Variable Background Active Contour model. The experimental results from the application of the proposed model on thyroid ultrasound images are apposed in Section III. Finally, in Section IV, the conclusions of this study are summarized.

\section{VARIABLE BACKGROUND ACTIVE CONTOUR MODEL}

\section{A. Active Contour Without Edges}

Active Contour Without Edges as posed in [7] has the form of a minimization problem: if we consider $\Omega$ as a bounded open subset of $R^{2}$ corresponding to image size, with $\partial \Omega$ the boundary, we seek for inf $F\left(c^{+}, c^{-}, C\right)$ :

$$
\begin{aligned}
F\left(c^{+}, c^{-}, C\right) & =\mu \cdot \operatorname{Length}(C) \\
& +\lambda^{+} \int_{\operatorname{inside(}(C)}\left|u_{0}(x, y)-c^{+}\right|^{2} d y d y \\
& +\lambda^{-} \int_{\text {outside }(C)}^{\mid}\left|u_{0}(x, y)-c^{-}\right|^{2} d x d y
\end{aligned}
$$

where $u_{0}: \Omega \rightarrow R$ is the given image, $C(s):[0,1] \rightarrow R^{2}$ a piecewise parameterized curve, $c^{+}$and $c^{-}$are unknown constants representing the average value of $u_{0}$ inside and outside the curve and parameters $\mu>0$ and $\lambda^{+}, \lambda^{-}>0$ are weights for the regularizing term and the fitting terms, respectively. This problem is a particular case of the minimal partition problem, for which the existence of minimizers has been proved in [11]. As in the minimum energy problem, the minimizer corresponds to the "equilibrium" of the regularizing and fitting terms that force the contour to stop.

In the level set method [10], $C \subset \Omega$ is represented by the zero level set of a Lipschitz function $\phi: \Omega \rightarrow R$, such that

$$
\begin{aligned}
& C=\partial \omega=\{(x, y) \in \Omega: \phi(x, y)=0\}, \\
& \text { inside }(C)=\{(x, y) \in \Omega: \phi(x, y)>0\}, \\
& \text { outside }(C)=\{(x, y) \in \phi(x, y)<0\}
\end{aligned}
$$

Using the Heaviside function $H$ and the one-dimensional Dirac measure $\delta$, defined respectively by

$$
\delta(z)=\frac{d}{d z} H(z), H(z)= \begin{cases}1, & \text { if } z \geq 0 \\ 0, & \text { if } z<0\end{cases}
$$

the constants $c^{+}$and $c^{-}$can be expressed as:

$$
\begin{gathered}
c^{+}(\phi)=\frac{\int_{\Omega} u_{0}(x, y) H(\phi(x, y)) d x d y}{\int_{\Omega} H(\phi(x, y)) d x d y} \\
c^{-}(\phi)=\frac{\int_{\Omega} u_{0}(x, y)(1-H(\phi(x, y))) d x d y}{\int_{\Omega}(1-H(\phi(x, y))) d x d y}
\end{gathered}
$$

Keeping $c^{+}$and $c^{-}$fixed, and minimizing $F$ with respect to $\phi$, we deduce the associated Euler-Langrange equation for $\phi$. For this purpose, we consider slightly regularized versions of $H$ and $\delta$, as described in [7]. Parameterizing the descent direction by an artificial time $t \geq 0$, the equation in $\phi(t, x, y)$ (with $\phi(0, x, y)=\phi_{0}(x, y)$ defining the initial contour) is

$$
\begin{gathered}
\frac{\partial \phi}{\partial t}=\delta(\phi)\left[\mu \cdot \operatorname{div}\left(\frac{\nabla \phi}{|\nabla \phi|}\right)-\lambda^{+}\left(u_{0}-c^{+}\right)^{2}+\lambda^{-}\left(u_{0}-c^{-}\right)^{2}\right]=0 \\
\phi(0, x, y)=\phi_{0}(x, y) \\
t \in(0, \infty),(x, y) \in \Omega
\end{gathered}
$$

In a practical implementation, a quantitative criterion should force the algorithm to stop when the changes of $\phi$ fall bellow a threshold for a fixed number of iterations. When this criterion is satisfied it is assumed that the minimizer is found and the corresponding equilibrium has been reached.

\section{B. Variable Background Active Contour}

Thyroid ultrasound images contain dark areas outside the thyroid gland and the model will tend to delineate all of them. This problem can be solved by using the function $\phi_{0}$ and the corresponding Heaviside function $H\left(\phi_{0}\right)$ to filter the part of the image outside the initial contour from the background and the foreground term, assuming that the user defines the initial contour in the interior of the thyroid gland.

In addition, inhomogeneities inside the thyroid gland may be present, increasing the background term in the model equation with an effect on the contour evolution. We consider a variable background area to reduce the inclusion of inhomogeneities. To achieve this, we introduce a difference term $\Delta(x, y)$ in the Active Contour Without Edges model:

$$
\Delta(x, y)=H(\phi(x, y)-a)-H(\phi(x, y)), \quad a>0
$$

and we require $\Delta(x, y)>0$ to restrict the background area. The constant $a$ determines the background area considered. This way it is ensured that when inhomogeneities inside the thyroid gland lie around a nodule, the background area changes to exclude them. This can be justified by the fact that the thyroid 
gland inhomogeneities cause an abrupt change of $\phi$ which results in $H(\phi(x, y)-a)=H(\phi(x, y))=1$ for the inhomogeneous areas in the image. Therefore the condition $\Delta(x, y)>0$ is not satisfied and these areas are excluded from the background. Introducing $H\left(\phi_{0}\right)$ and $\Delta(x, y)$ in Eq. (5), we derive:

$$
c^{-}(\phi)=\frac{\int_{\Omega} u_{0}(x, y)(1-H(\phi(x, y))) H\left(\phi_{0}(x, y)\right) \Delta(x, y) d x d y}{\int_{\Omega}(1-H(\phi(x, y))) H\left(\phi_{0}(x, y)\right) \Delta(x, y) d x d y}
$$

The above equations describe the proposed Variable Background Active Contour model.

\section{RESULTS}

Thyroid ultrasound examinations were performed on 24 patients using a digital ultrasound system HDI 3000 ATL with a 5-12 MHz linear transducer. The acquired digital images had a resolution of $256 \times 256$ pixels and 256 gray-level depth.

We applied the Active Contour Without Edges model and the Variable Background Active Contour model for the detection of thyroid nodules in the acquired ultrasound images. For the purposes of our study we adopted the image intensity as the supervising feature for the contour evolution, to enable the detection of hypo-echoic thyroid nodules. Low echogenicity characterizes the majority of thyroid nodules and especially those that are suspect of malignancy [12]. The model constants used in the experiments are derived empirically as $\lambda^{+}=5, \lambda^{-}=5, \mu=650$ and $a=10^{-13}$.

A special purpose software suite implementing these algorithms was developed on Microsoft Visual $\mathrm{C}++$.

An expert radiologist manually delineated the thyroid nodules to enable comparisons with the active contour models. As a measure of similarity between the area inside the active contour delineation $A$ and the area inside the expert's delineation $\mathrm{B}$, we use the overlap value [5]:

$$
i=\frac{A \cap B}{A \cup B}
$$

For a perfect matching $i$ is expected to be equal to 1 .

Figure 1 illustrates the average overlap value achieved by the two models. The Active Contour Without Edges model achieved $i=0.86 \pm 0.04$ while the proposed Variable Background Active Contour Model achieved $i=0.94 \pm 0.01$.

Indicative results for two thyroid ultrasound images are illustrated in Fig. 2. The first image (Fig.2a) contains one nodule while the second (Fig.2b) contains two nodules. It can be observed that both algorithms have detected the nodules. The delineation achieved by the Variable Background Active Contour model is more similar to the expert's delineation compared to the one achieved by the Active Contour Without
Edges model. Moreover, for the two-nodule case the proposed model managed to produce separate contours while the Active Contour Without Edges model failed to do so.

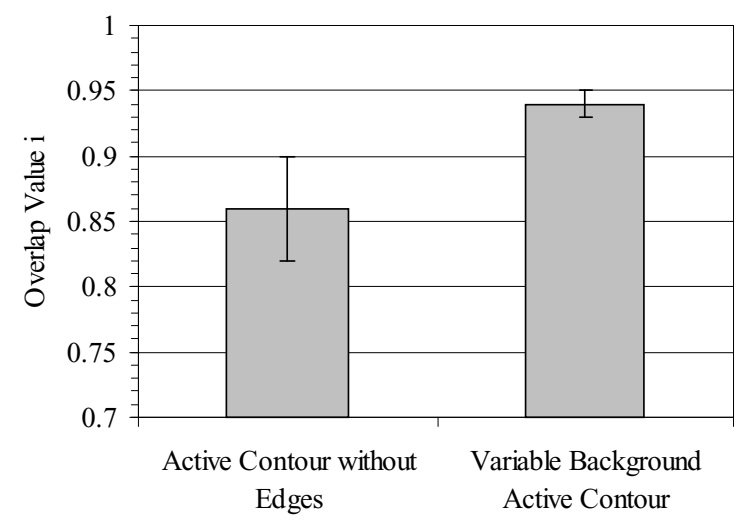

Fig. 1. Average overlap values achieved by the two active contour models.

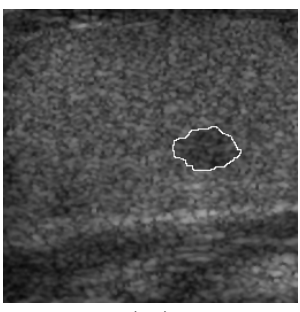

$\left(a_{1}\right)$

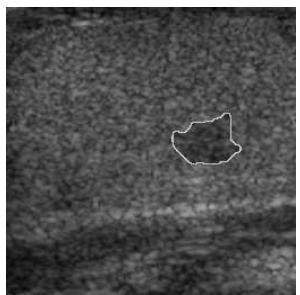

$\left(a_{2}\right)$

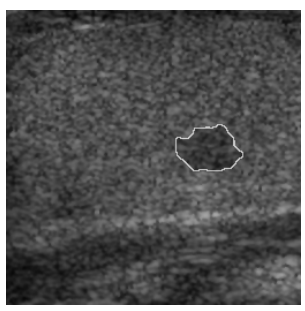

$\left(a_{3}\right)$

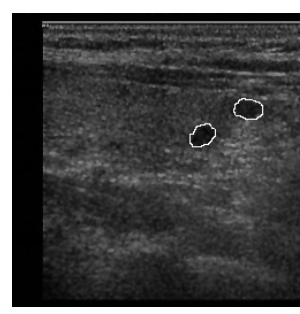

$\left(b_{1}\right)$

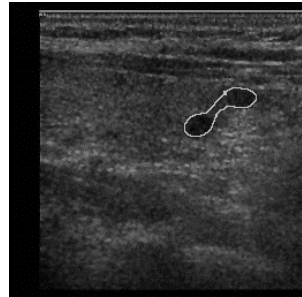

$\left(b_{2}\right)$

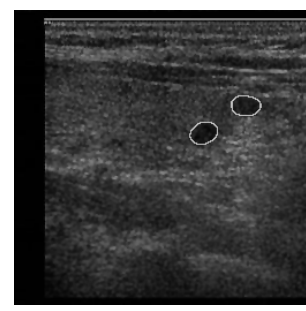

$\left(b_{3}\right)$
Fig. 2. Two example thyroid ultrasound images: $\left(a_{1}-b_{1}\right)$ Contours delineated by the expert radiologist, $\left(a_{2}-b_{2}\right)$ Active Contour Without Edges, $\left(\mathrm{a}_{3}-\mathrm{b}_{3}\right)$ Variable Background Active Contour.

\section{CONCLUSION}

We have proposed a Variable Background Active Contour model and applied it for the detection of thyroid nodules in ultrasound images. In this model, the background is a variable subset of the image, which changes shape to reduce the effects 
of background inhomogeneity. The results of the experimental study lead to the following conclusions:

1) The proposed model can be applied without preprocessing due to its edge independency.

2) It is capable of detecting two or more nodules taking advantage of its ability for topological changes such as contour splitting.

3) It provides improved accuracy compared to the Active Contour Without Edges model. In particular, the improvement in contour accuracy is important due to the fact that nodule size and shape are factors affecting medical expert's characterization [12].

Future perspectives of this work include the incorporation of an object's inhomogeneity as well as the embedment of textural features [13] to supervise contour evolution, which could enable the detection of non hypo-echoic nodules.

\section{ACKNOWLEDGMENT}

This work was partially funded by the Special Account of Research Grants, National and Kapodestrian University of Athens.

\section{REFERENCES}

[1] S. Feld et all, "AACE Clinical Practice Guidelines for the Diagnosis and Management of Thyroid Nodules", Endocrine Practice, vol. 2, pp. 7884, January/February 1996.

[2] S.V.B. Jardim, M.A.T. Figueiredo, "Automatic Contour Estimation In Fetal Ultrasound Images”, Proc. ICIP’03, 2003.
[3] R. Adams, L. Bischof, "Seeded Region Growing", IEEE Transactions on Pattern Analysis and Machine Intelligence, vol. 16, no. 6, pp. 641-647, June 1994.

[4] R. Pohle, K. Toennies, "Segmentation of Medical Images using Adaptive Region Growing”, Proc. SPIE (Medical Imaging 2001), 4322, 2001.

[5] X. Hao, C. Bruce, C. Pislaru, and J.F. Greenleaf, "A Novel Region Growing Method for segmenting ultrasound images”, IEEE International Ultrasonics Symposium, October 2000.

[6] A. Blake, M. Isard, "Active Contours: The Application of Techniques from Graphics, Vision, Control Theory and Statistics to Visual Tracking of Shapes in Motion", Springer Verlag, 1999.

[7] T.F. Chan, L.A. Vesse, "Active Contours Without Edges", IEEE Trans. Image Processing, vol. 7, pp. 266-277, February 2001.

[8] S.C. Zhu, A.L. Yuille, "Region competition: Unifying Snakes, Region Growing, and Bayes/MDL for Multi-Band Image Segmentation”, IEEE Trans. On Pattern Analysis and Machine Intelligence, vol. 18, no.9, pp. 884-900, September 1996.

[9] N. Paragios, R. Deriche, "Geodesic Active Contours for Supervised Texture Segmentation”, proc. Computer Vision and Pattern Recognition 1999, pp. 2422-2427, 1999.

[10] S. Osher, J. Sethian, "Fronts Propagating with Curvature- Dependent Speed: Algorithms Based on the Hamilton-Jacobi Formulations", Journal Of Computational Physics, vol. 79, pp. 12-49, 1988.

[11] D. Mumford, J. Shah, "Optimal Approximation by Piecewise Smooth Functions and Associated Variational Problems", Commun. Pure Appl. Math., vol. 42, pp. 577-685, 1989.

[12] E. Papini et al, "Risk of Malignancy in Nonpalpable Thyroid Nodules: Predictive Value of Ultrasound and Color-Doppler Features", The Journal of Clinical Endocrinology \& Metabolism, vol. 87(5), pp. 19411946, 2002.

[13] S. Theodoridis, K. Koutroumbas, "Pattern Recognition", Academic Press, 1998 